\title{
Stem cells as a potential therapeutic trend for experimental trichinosis
}

\author{
Original \\ Article \\ Mohamed H Sarhan', Samia E Etewa ${ }^{1}$, Abd-Allah A Al-Hoot ${ }^{2}$, Salwa Z Arafa ${ }^{2}$, \\ Rodyna AMA Shokir ${ }^{2}$, Howayda SF Moawad ${ }^{1}$, Samira M Mohammad ${ }^{1}$
}

Departments of Medical Parasitology ${ }^{1}$ and Zoology ${ }^{2}$, Faculties of Medicine ${ }^{1}$ and Science ${ }^{2}$, Zagazig University, Zagazig 44519, Egypt

\begin{abstract}
Background: Trichinella spiralis causes trichinosis through ingestion of pork contaminated by its infective larvae, resulting in intestinal and muscular phases of infection in the same host. Stem cells (SCs) treat some diseases due to their capacity for trans-differentiation and immunomodulation.

Objective: To assess the therapeutic impact of mesenchymal stem cells (MSCs) during intestinal and muscular stages in T. spiralis-experimentally infected mice; used alone as monotherapy or combined with mebendazole (MBZ).

Material and Methods: Forty albino mice were used to obtain bone marrow MSCs. Another 100 albino mice were divided into 2 groups 50 mice each simulating intestinal (a) and muscular (b) phases. Each group was further subdivided into 5 subgroups, 10 mice each as follows: G1a and G1b: negative non infected control; G2a and G2b: positive infected control; G3a and G3b: infected and MBZ treated; G4a and G4b: infected and MSCs tested; and G5a and G5b: infected and combined MBZ and MSCs therapy tested. Mice of intestinal phase were sacrificed on $7^{\text {th }}$ day post-infection (PI) while mice of muscular phase were sacrificed on $49^{\text {th }}$ day PI. Assessment was done by parasitological assessment (the number of adult worms in the intestine in groups (a) and the number of encysted larvae in the diaphragm in groups (b); histopathological and histochemical assessment of all groups using hematoxylin and eosin (H\&E) and Feulgen stains of different mice tissues and ultrastructural assessment using transmission electron microscope (TEM).

Results: The combined therapy was potent; it showed the highest significant reduction in the number of intestinal worms and encysted muscular larvae with preservation of the different tissues elements as investigated by different stains and TEM.

Conclusion: MSCs can be used as additive/synergistic therapy in the treatment of trichinosis.
\end{abstract}

Keywords: apoptosis; mebendazole; mesenchymal stem cells; transmission electron microscope; T. spiralis.

Received: 26 April, 2021, Accepted: 22 June, 2021.

Corresponding Author: Mohamed H. Sarhan, Tel.: +20 1020260608, E-mail: drsarhan@zu.edu.eg

Print ISSN: 1687-7942, Online ISSN: 2090-2646, Vol. 14, No. 2, August, 2021.

\section{INTRODUCTION}

Trichinosis has a wide distribution all over the world. It infects nearly 10,000 individuals yearly with a mortality rate of $0.2 \%{ }^{[1]}$. It is caused by ingestion of the encysted larvae of the nematode genus Trichinella in raw or undercooked pork ${ }^{[2]}$. T. spiralis species is the most virulent species and is the pathogenic one to $\operatorname{man}^{[3]}$. In human host, trichinosis has two phases, an intestinal and a muscular phase. After ingestion of infected undercooked meat, the first larval stages are released by gastric digestion, reach the intestine, and develop into adult worms. After mating, adult females shed larvae that migrate through lympho-vascular system to other organs. Predicted larvae invade skeletal muscles forming nurse cell complexes that initiate an inflammatory reaction in muscular tissues and other organs causing manifestations as high fever, diarrhea, myalgia, and periorbital edema ${ }^{[2]}$. Severe complications as myocarditis may precipitate death; lung and central nervous system may be affected ${ }^{[4]}$.

The specific anthelminthic, MBZ, is a synthetic broad-spectrum benzimidazole anthelminthic, and is the primary drug used for trichinosis treatment ${ }^{[2]}$. It acts through selective binding to the parasite monomer, beta-tubulin, while it has a minute influence on that of the host resulting in microtubule polymerization inhibition ${ }^{[5]}$. Disturbance of cytoplasmic microtubules results in blocking the uptake of glucose and other nutrients, leading to gradual immobilization and subsequent death of the helminths ${ }^{[6]}$. However, it has limited bioavailability; and a high degree of resistance by human hosts was recorded ${ }^{[7]}$, with a restricted impact on the encapsulated muscle larvae ${ }^{[8]}$. Therefore, there is need to search for new 
substitutes/synergistic with better bioavailability to cross the different biological barriers and tolerate the host immune response; e.g., SCs treatment.

It is worth mentioning that SCs have the capacity for indefinite discrimination into several specialized, diverse cells in the body during life ${ }^{[9]}$. Moreover, they show plasticity or trans-differentiation ${ }^{[10]}$. Through SCs treatment, new cells can be introduced into injured sites aiding in the management of several diseases as cancers, diabetes mellitus, parkinsonism, neurological, cardiovascular disorders and many other diseases ${ }^{[11]}$. Lately, SCs have improved some parasitic diseases as $S$. mansoni through interleukin-10 production and immunomodulation ${ }^{[8]}$. They were used to treat malaria, leishmaniasis and trypanosomiasis by improving the function of the tissues of the involved organs ${ }^{[12]}$. Moreover, it was reported that the injured liver tissue caused by hydatid cyst can be restored without complete fading of the cyst by using SCs after treatment with albendazole as SCs can modify the protective immune reactions produced by the host ${ }^{[13]}$. So, these results motivated clinical trials in humans. In this work, we aimed to evaluate the impact of bone marrow MSCs therapy on experimental trichinosis in murine models.

\section{MATERIAL AND METHODS}

This experimental study lasted from June 2017 to May 2018. It was conducted in the postgraduate research laboratory of Medical Parasitology Department and Clinical Chemistry and Stem Cells laboratories of Biochemistry Department, Faculty of Medicine in co-operation with Zoology Department, Faculty of Science, Zagazig University, Sharkia, Egypt.

Experimental animals and models: The study was performed on 140 parasitic free, male, BALB/c mice, 25-30 gm weight and aging 6-8 weeks. They were obtained from Theodore Bilharz Research Institute, Giza, Egypt, and were kept under suitable temperature and light in addition to the standard food and water provisions. For evaluation of intestinal (50 mice) and muscular stages (50 mice) of infection, a total of eighty mice were infected orally with $\sim 200 \mathrm{~T}$. spiralis larvae/ mouse; 20 were kept as healthy non infected controls. The remaining 40 mice were retained for procuring bone marrow MSCs.

Parasite and infection: T. spiralis strain was extracted from infected pig diaphragm muscles gained from El-Bassatine Abattoir, Cairo. It was preserved by consecutive passages through mice in the Animal House of the Faculty of Medicine, Zagazig University, Sharkia, Egypt according to Gamble ${ }^{[14]}$. Heavily infected pigs' diaphragms were minced and digested in $1 \%$ pepsin-hydrochloride overnight at $37^{\circ} \mathrm{C}$. Free larvae were collected by sedimentation, and washed many times in physiological saline $0.85 \%$. Live larvae $/ \mathrm{ml}$ were counted $^{[15]}$ and 80 mice were each infected orally with $\sim 200$ motile and coiled larvae ${ }^{[16]}$.

Study design: In this work, one hundred male albino mice were divided into 2 groups (G) of 50 mice each, simulating intestinal (a) and muscular (b) phases. Each group was further subdivided into 5 subgroups, 10 mice each: G1a and G1b as negative controls, G2a and G2b as infected non-treated controls, G3a and G3b infected then MBZ treated, G4a and G4b infected then MSCs tested and G5a and G5b infected then received both MSCs and MBZ.

For assessment during the intestinal phase, MBZ (50 mg/ kg body weight) and MSCs (total of 1,000,000 MSCs in three divided doses) were orally administered beginning from the second day PI for three consecutive days. Mice of G1a, G2a, G3a, G4a and G5a were sacrificed $7^{\text {th }}$ day PI. During the muscular phase, mice of G3b were given MBZ ( $50 \mathrm{mg} / \mathrm{kg}$ body weight) orally and those in G4b were given MSCs (total of 1,000,000 MSCs in three divided doses) by intraperitoneal (IP) and intramuscular (IM) injection using $1 \mathrm{~cm}$ length insulin syringe. Mice of G5b received MSCs by IP and IM routes in combined therapy with oral MBZ. Therapies were given on three successive days starting $14^{\text {th }}$ day PI. Mice of G1b, G2b, G3b, G4b and G5b were sacrificed $49^{\text {th }}$ day PI.

For parasitological, histopathological, and histochemical assessments, intestinal specimens were collected from mice sacrificed on the $7^{\text {th }}$ day PI; whereas muscular specimens were collected from mice sacrificed on the $49^{\text {th }}$ day PI. Diaphragms from mice sacrificed on the $49^{\text {th }}$ day PI were examined using TEM.

Preparation of MSCs: MSCs were collected by flushing 40 Swiss albino male mice femurs and tibias ${ }^{[17]}$. Testing of the viability of SCs was by trypan blue stain, where transparent cells which did not take blue color were considered viable ${ }^{[18]}$. By the inverted microscope, cultured live cells appeared adhesive and fusiform ${ }^{[18]}$. Also, they exhibited positive CD105 and negative CD34 surface markers expression when analyzed by flow cytometry according to Calabrò et al. ${ }^{[19]}$ in the laboratory of Clinical Pathology Department, Faculty of Medicine, Zagazig University. Trypsin $0.25 \%$ was used to detach the second passage of SCs by centrifugation for $5 \mathrm{~min}$ at $2000 \mathrm{rpm}$ followed by washing in phosphate buffer saline. Cells were then incubated with monoclonal antibody labeled with Endoglin-Phycoerythrin as a link, to reveal CD105. Unbound antibody was washed from the cells followed by fluorescein staining. The staining strength was directly proportional to the CD105 protein density. Flow cytometer analysis at $488 \mathrm{~nm}$ wavelength laser excitation was used to measure the CD105 cell surface expression ${ }^{[20]}$. According to Louis and Siegel ${ }^{[21]}$, viable cells were counted by a hemocytometer and calculated where the total number of viable cells $/ \mathrm{ml}$ $=$ the number of viable cells counted/square X 10,000 
dilution factor; viable cells total number in a sample $=$ total number of viable cells $/ \mathrm{ml} \mathrm{X}$ sample volume in $\mathrm{ml}$. Accordingly, a dose of 1,000,000 MSCs/mouse was given either orally (intestinal phase) or parenterally (muscular phase) in three divided doses ${ }^{[22]}$.

Homing of SCs in tissues: SCs were labeled with fluorescent PKH26 stain (Sigma, Aldrich, Egypt) then visualized by a fluorescent microscope according to $\mathrm{Li}$ et $a l_{.}^{[23]}$. It was reported that PKH26 is an excellent cell linker dye for in vivo cells-following studies, precisely when labeled cells need to be followed for long periods because of its highly stable fluorescence.

Drug (MBZ) form and dose: The drug was purchased from Pharco Co. (Cairo, Egypt) in $30 \mathrm{ml}$ suspension $(100 \mathrm{mg} / 5 \mathrm{ml})$. It was administered orally using a $1 \mathrm{ml}$ syringe equipped with a blunt, curved, 18-gauge needle for 3 successive days at a $50 \mathrm{mg} / \mathrm{kg}$ body weight dose.

\section{Parasitological assessment}

Intestinal adult worms count ${ }^{[15]}$ : The small intestine was entirely divided into 4 equal lengths, each part was opened, washed and held for $2 \mathrm{~h}$ in saline to permit exit of worms, then followed by washing for many times until the fluid became transparent. Worm suspension was then centrifuged for $5 \mathrm{~min}$ at $1500 \mathrm{rpm}$ to sediment the worms. Worm burden was counted on a dissecting microscope.

Muscular encysted larvae count ${ }^{[24]}$ : Proper dissection of the whole diaphragm was followed by digestion then centrifugation to sediment the larvae. Thereafter, viable larva burden (i.e., motile, and coiled) was counted in each mouse's diaphragm using McMaster Counting Slide.

Assessment of therapeutic effects: Estimation was through counting the mean number of live worms (G2aG5a) and that of viable larvae (G2b-G5b). Drug efficacy $(\%)=[(A-B) / A] \times 100$, where $A=$ the counted worms or larvae of infected control mice, and $\mathrm{B}=$ the counted worms or larvae from tested mice ${ }^{[3]}$.

Histopathological assessment: Intestinal specimens $(\sim 1 \mathrm{~cm})$ were cut at the junction of the proximal $1 / 3$ and distal 2/3, from mice sacrificed on the $7^{\text {th }}$ day $\mathrm{PI}^{[25]}$. Muscle specimens were collected from mice sacrificed on the $49^{\text {th }}$ day PI from the diaphragm, tongue, and hind legs ${ }^{[26,27]}$. The collected specimens were fixed in $10 \%$ formalin. dehydrated in ascending grades of alcohol, cleared in xylol, implanted in blocks of paraffin, sectioned with $5 \mu$ thickness, stained by hematoxylin and eosin (H\&E) and examined microscopically ${ }^{[28]}$. Inflammatory grading was recorded as: score 0 indicated no lesion, while scores 1-4 indicated minimal, mild, moderate, and severe lesions, respectively ${ }^{[29]}$.

Histochemical assessment: Feulgen stain was used to detect alterations resulting from hydrolysis of nuclear DNA acid due to apoptosis. Stained tissues were carefully fixed avoiding strong acid utilization to prevent unnecessary de-staining. Ordinarily, DNA stains bright red, and the background counterstains green. Changes of nuclear DNA coloration were used to classify apoptosis as: light red, slight red, and less bright red representing extreme, moderate, and mild apoptosis, respectively. In contrast, bright red nuclei represent regular nuclei ${ }^{[30]}$.

Ultrastructural assessment: Sections of diaphragm specimens collected from sacrificed mice on $49^{\text {th }}$ day PI, were fixed for $20 \mathrm{~h}$ in a mixture of $2.5 \%$ glutaraldehyde, $2 \%$ paraformaldehyde and cacodylate buffer $0.1 \mathrm{M}$ (pH 7.4), and post-fixed in 1\% osmium tetroxide (OsO4, Sigma-Aldrich) and $0.8 \%$ potassium ferrocyanide (K4FeCN6, Sigma-Aldrich). The samples were dehydrated in ethanol, permeated with propylene oxide then embedded in Spurr resin. Ultrathin $\sim 50 \mathrm{~nm}$ sections were then examined by TEM (JEM 1200 EX), to inspect and observe the nurse cell-larva complex and surrounding tissue reaction ${ }^{[31]}$.

Statistical analysis: The collected data were evaluated using Statistical Package for Social Sciences (SPSS), windows version 20 . Student $(t)$ test and analysis of variance (ANOVA) test were utilized to determine probable differences among the sample groups. The $P$ value $<0.05$ was considered significant.

Ethics approval: Approval was obtained from the Institutional Animal Care and Use Committee of Zagazig University (IACUC-ZU). The procedures used in this study adhered to the principles of the Declaration of Helsinki.

\section{RESULTS}

Parasitological results of the intestinal phase: Tables (1 and 2) showed a significant reduction in the mean number of adult $T$. spiralis worms in G5a (combined therapy) followed by G3a (MBZ alone) with percentage reductions of $85.6 \%, 84.5 \%$ respectively. In contrast, the insignificant decline was noticed in G4a (MSCs alone) with $2.4 \%$ percentage reduction relative to infected control G2a.

Parasitological results of the muscular phase: Tables (3 and 4) showed that combined therapy (G5b) was the most successful treatment with significant decrease in T. spiralis larvae number followed by G3b (MBZ only) then G4b (MSCs only) with percentage reductions of $91.7 \%, 87.3 \%$ and $70.0 \%$, respectively.

Histopathological reactions of the intestinal phase: While G1a showed no pathological alterations, variable histopathological observations were recorded in infected mice ranging from mild inflammatory reaction with affection of villi (G3a), and areas of hemorrhage 
PARASITOLOGISTS UNITED JOURNAL

Table 1. The mean number of adult worms of T. spiralis in the intestine in both control and tested groups.

\begin{tabular}{|c|c|c|c|}
\hline \multirow{2}{*}{ Study groups } & \multicolumn{3}{|c|}{ Adult T. spiralis worm/mice } \\
\hline & Mean \pm SD & Range & Reduction \% \\
\hline Group 1a (Negative control) & $0 \pm 0$ & -- & -- \\
\hline Group 2a (Positive control) & $119.4 \pm 38$ & $70-180$ & -- \\
\hline Group 3a (MBZ treated) & $18.2 \pm 4.3$ & $10-25$ & 84.5 \\
\hline Group 4a (MSCs treated) & $116.5 \pm 33.7$ & $75-170$ & 2.4 \\
\hline Group 5a (MBZ+MSCs treated) & $17.3 \pm 4.99$ & $13-30$ & 85.5 \\
\hline$F$-test ANOVA & \multicolumn{3}{|c|}{$\boldsymbol{F}: 80.575$, critical $F$ value: $2.9604, \boldsymbol{P}<\mathbf{0 . 0 0 1}$} \\
\hline
\end{tabular}

SD: Standard deviation.

Table 2. Least significant difference (LSD) test for comparison of mean number of adult worms of T. spiralis/mice between control and other studied groups.

\begin{tabular}{lccc}
\hline \hline & Group 2a (Positive control) & Group 3a (MBZ treated) & Group 4a (MSCs trated) \\
\hline Group 5a (MBZ+MSCs treated) & $<\mathbf{0 . 0 0 1 *}$ & $>0.05(\mathrm{NS})$ & $<\mathbf{0 . 0 0 1}^{*}$ \\
Group 4a (MSCs treated) & $>0.05(\mathrm{NS})$ & $<\mathbf{0 . 0 0 1 *}$ & \\
Group 3a (MBZ treated) & $<\mathbf{0 . 0 0 1 *}$ & & \\
\hline \hline
\end{tabular}

*: Significant; NS: Not significant.

Table 3. The mean number of T. spiralis encysted larvae in the muscular phase in both control group and tested groups.

\begin{tabular}{|c|c|c|c|}
\hline \multirow{2}{*}{ Study groups } & \multicolumn{3}{|c|}{ T. spiralis larvae/mice } \\
\hline & Mean \pm SD & Range & Reduction $\%$ \\
\hline Group 1b (Negative control) & $0 \pm 0$ & -- & -- \\
\hline Group 2b (Positive control) & $1933.4 \pm 841.7$ & $850-3120$ & -- \\
\hline Group 3b (MBZ treated) & $244.1 \pm 106.1$ & $100-400$ & 87.3 \\
\hline Group 4b (MSCs treated) & $578.8 \pm 250.1$ & $200-920$ & 70 \\
\hline Group 5b (MBZ+MSCs treated) & $161.0 \pm 97.5$ & $70-350$ & 91.7 \\
\hline F-test ANOVA & \multicolumn{3}{|c|}{$\boldsymbol{F}: 44.61$, critical $F$ value: $2.960, \boldsymbol{P}<\mathbf{0 . 0 0 1}$} \\
\hline
\end{tabular}

SD: Standard deviation.

Table 4. Least significant difference (LSD) test for comparison of mean number of encysted T. spiralis larvae between control group and other studied groups.

\begin{tabular}{lccc}
\hline \hline & Group 2b (Positive control) & Group 3b (MBZ treated) & Group 4b (MSCs trated) \\
\hline Group 5b (MBZ+MSCs treated) & $<0.001^{*}$ & $>0.05(\mathrm{NS})$ & $<0.05^{*}$ \\
Group 4b (MSCs treated) & $<0.001^{*}$ & $<0.05^{*}$ & \\
Group 3b (MBZ treated) & $<0.001^{*}$ & & \\
\hline \hline
\end{tabular}

*: Significant; NS: Not significant.

(G4a). On the other hand, combined therapy (G5a) showed obvious drop in inflammatory reaction with intact villous structure in comparison with infected non-treated group (G2a) (Fig. 1 A-E).

Histopathological results of the muscular phase: Similarly, G1b showed no histopathological variations, G2b showed severe inflammation with large number of encysted larvae, G3b showed cracked vacuolated capsules, G4b showed mild inflammation, and G5b showed disintegrated larvae with destroyed capsules (Fig. 2 A-E).

Histochemical reactions in the intestinal phase: No histochemical changes were observed in cut sections of small intestine of G1a. Severe apoptosis was observed in G2a and G4a, while G3a showed mild apoptosis evidenced by the faint red coloration of the cellular nuclei of the intestinal mucosa and the different layers of the intestinal wall. With dual therapy, G5a showed normal circular shape of cellular nuclei of the intestinal mucosa and the different layers of the intestinal wall (Fig. 3 A-E).

Histochemical reactions in the muscular phase: No histochemical changes were observed in GIb as compared to severe apoptosis in Gb2. Moderate apoptosis was detected in G3b, while G4b and G5b showed mild apoptosis (Fig. 4 A-E).

Ultrastructural reactions: TEM study aimed to detect and distinguish the ultrastructural changes in the nurse cell-larva complex inside the diaphragms of the different studied groups. Mice of non-infected group (G1b) showed no ultrastructural changes (Fig. 5). Those infected and non-treated (G2b) showed intact spiral larvae surrounded by nurse cells with alteration of muscle fiber matrix encircling the larvae 


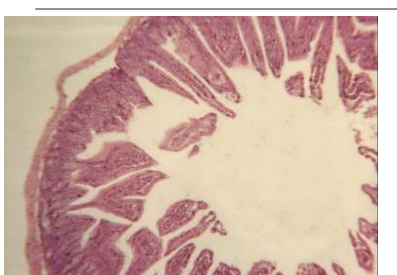

(A)

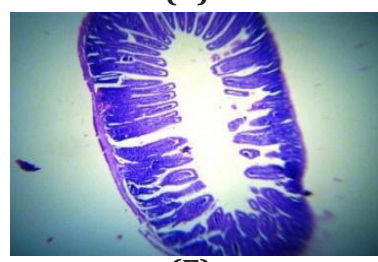

(E)

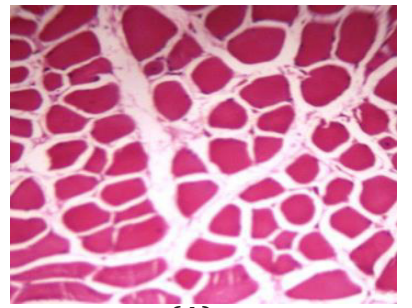

(A)

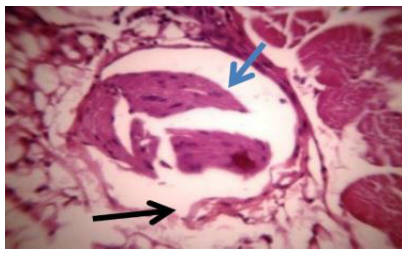

(E)

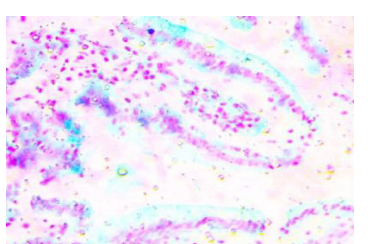

(A)

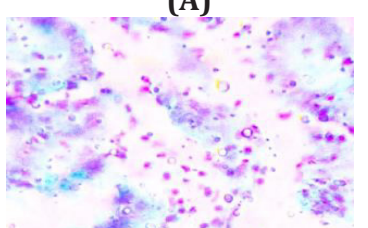

(E)

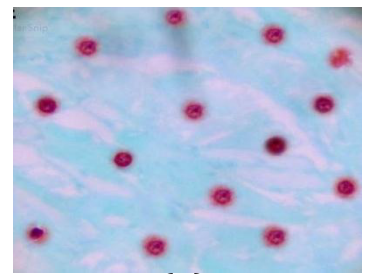

(A)

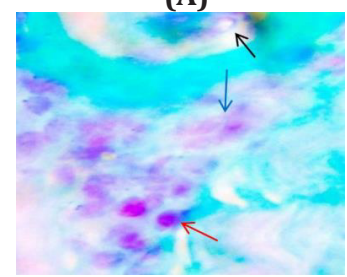

(E)

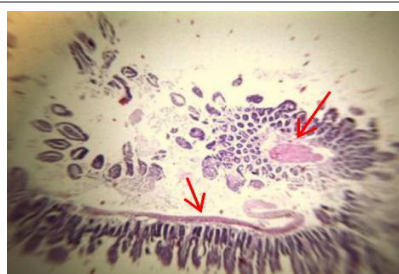

(B)

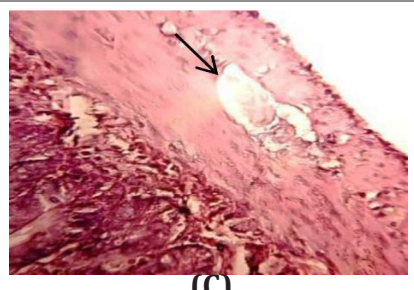

(C)

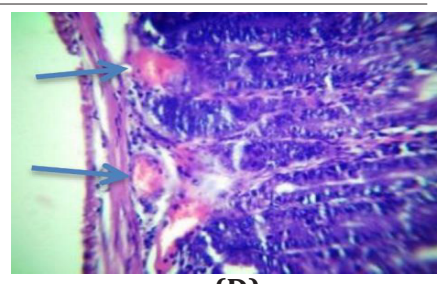

(D)
Fig. 1. Sections of the small intestine stained with H\&E from the studied groups on $7^{\text {th }}$ day PI. A: No histopathological alterations were detected in the sections of non-infected control G1a (400X). B: Intestinal sections of infected G2a showing cut section of adult worm detected in the lumen of the small intestine (red arrows) (100X). C: Section of MBZ-tested G3a showing adult worms (black arrow) inside the intestine wall with mild inflammation, villus atrophy and degeneration (400X). D: Section of MSCs tested G4a showed areas of hemorrhage scattered among the layers of the intestinal wall (blue arrows) up to the musculosa due to adult worm penetration of the intestinal wall (400X). E: In G5a (MBZ + MSCs), there were no adult worms, villi were intact, no abnormality was detected either in the lumen or within the intestine wall (100X).

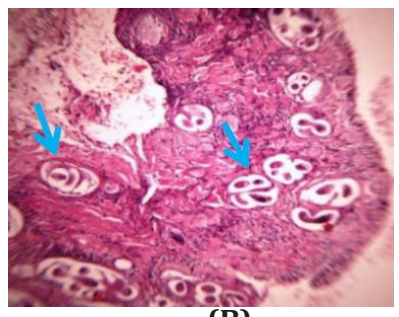

(B)

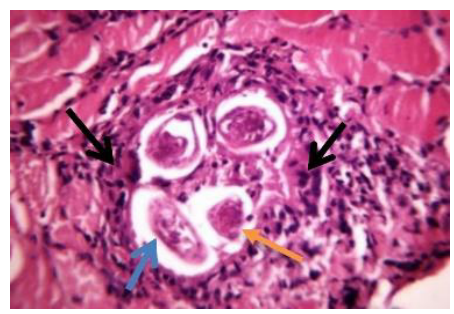

(C)

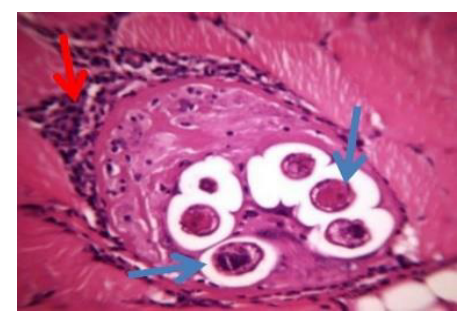

(D)
Fig. 2. Sections of skeletal muscles stained with H\&E of the studies groups on the $49^{\text {th }}$ day PI. A: No histopathological variations were noticed in sections of non-infected control G1b (400X). B: Infected control G2b showed massive number of encysted larvae of $T$. spiralis (blue arrows) with severe infiltration (100X). C: MBZ treated G3b showed homogenized larvae (blue arrow), vacuolated capsules, cracked into thin layers. Intense inflammatory cellular diffusion (black arrows) encircle the capsule and penetrate it, even adhering to the larva (orange arrow) (400X). D: MSCs tested G4b displayed encysted larvae (blue arrows) encircled by dense intact capsule with mild inflammation and granulation tissue infiltrate (red arrow) (400X). E: MBZ + MSCs tested G5b showed disintegrated T. spiralis larvae (blue arrow), incomplete destruction of the capsule (black arrow) with encircling and penetrating inflammatory cells (400X).

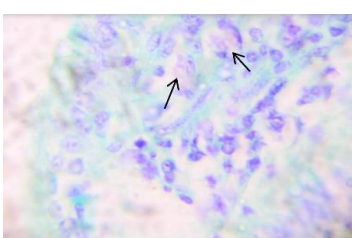

(B)

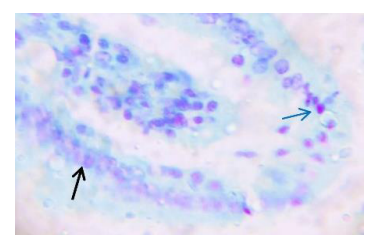

(C)

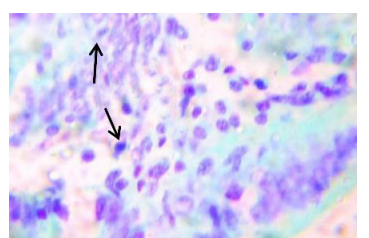

(D)
Fig. 3. Intestinal sections of the studied groups stained by Feulgen stain on $7^{\text {th }}$ day PI (1000X). A: G1a showed no histochemical changes with appearance of brightly red normal nuclei of enterocytes. B: Control infected G2a showed severe apoptosis (black arrows). C: MBZ tested G3a showed mild apoptosis (black arrow) and areas of affected nuclei mixed with standard rounded nuclei (blue arrow) of intestinal mucosa and the different layers of the intestinal wall. D: MSCs tested G4a showed severe apoptosis (black arrows). E: MBZ + MSCs tested G5a showed red coloration and normal circular shape of cellular nuclei of the intestinal mucosa and the different layers of the intestinal wall.

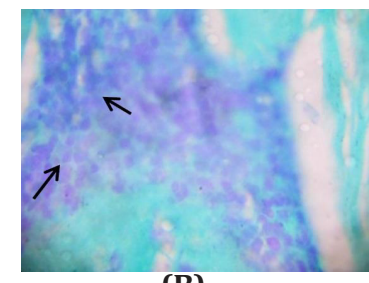

(B)

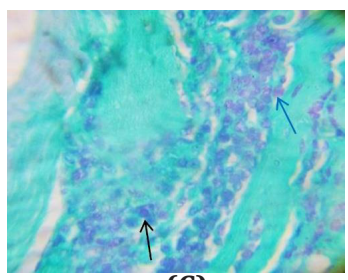

(C)

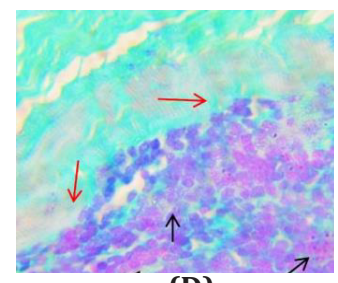

(D)

Fig. 4. Skeletal muscle sections of the studies groups stained by Feulgen stain on $49^{\text {th }}$ day PI (X1000). A: Non-infected G1b showed no histochemical changes. B: Faint red nuclei of muscle (black arrows) indicating severe apoptosis in infected G2b. C: MBZ treated G3b showed areas of affected nuclei of myocytes (blue arrow) mixed with nearly normal myocytes nuclei (black arrow); moderate apoptosis was detected by light red coloration of the myocytes nuclei. D: MSCs tested G4b showed mild apoptosis (faint red coloration of nuclei of myocytes), mild ballooning (black arrow), and intact myocytes nuclei (red arrows). E: MBZ + MSCs tested group showed mild apoptosis (blue arrow) and faint red coloration of the myocytes nuclei (red arrow) surround the encysted larvae (black arrow). 
(Fig. 6 A-D). Mice receiving MBZ alone (G3b) showed calcification in the larval cuticle with reduced glycogen content and appearance of mononuclear phagocytes and eosinophils around the capsular layer (Fig. $7 \mathrm{~A}$, B). Those that received MSCs (G4b) showed marked vacuolization with glycogen loss in the majority of larvae with various degeneration signs and calcification (Fig. 8 A-E). Finally, combined therapy (G5b) showed normal ultrastructural organization of muscle cells with the appearance of healing stage (restoration of the normal muscle fibers) and mononuclear phagocytes (Fig. 9 A, B).

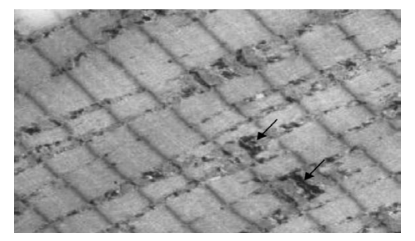

Fig. 5. TEM ultra-micrograph of diaphragm of healthy control G1b showing no ultrastructural alterations. Nuclei presented a quiescent feature with tiny, slender, commonly well united sarcomeres, mitochondria, and small areas of thick glycogen granules in between (black arrows) (X5100)

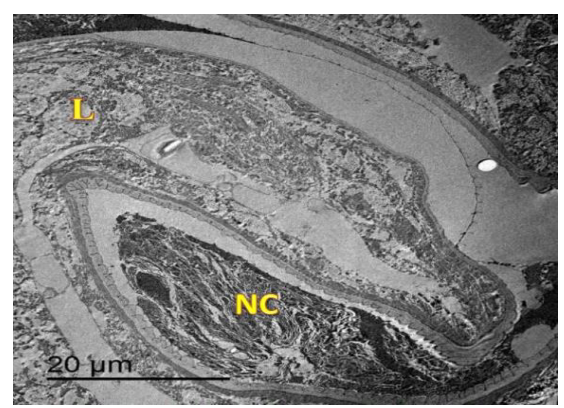

(A)

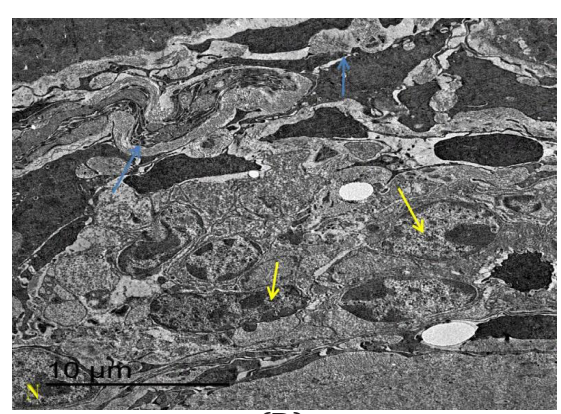

(D)

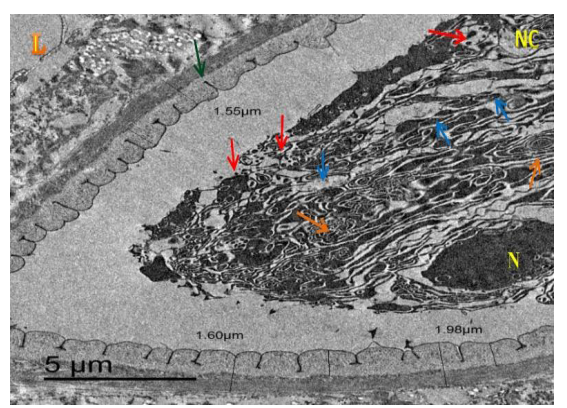

(B)

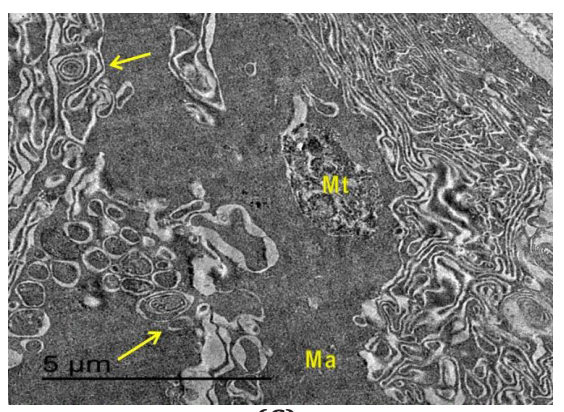

(C)

Fig. 6. TEM ultra-micrograph of diaphragm of T. spiralis infected control G2b. A: Intact spiral larva (L), surrounded by nurse cell (NC), without alterations of larval shape (X5100). B: Details of the nurse cell area (NC) in direct connection to larva (L). A large amount of smooth and rough reticulum (orange arrows), vesicles, and Golgi complexes, hypertrophic nuclei (N) and excessive mitochondrial numbers were detected (blue arrows). Near the larva surface is a bilayer membrane resembling the classic "unit membrane" described as a railroad track with two thick lines nearly $1.98 \mu \mathrm{m}$ in thickness (green arrow) with an apparent space in between. Glycogen particles were detected in big amount (red arrows) (X17000). C: Alteration of muscle fiber matrix (Ma) encircling the larva (L) and enclosing a massive quantity of highly expanded and disordered lamellar cytoplasm network (yellow arrows) with swollen mitochondria (Mt) (X25500). D: Details in nurse cell-larvae complex showed that the structural integrity of sarcomeres was lost, with increased hypertrophy and hyperplasia of mitochondria (yellow arrows), rise in the nuclei (N) number and size and a distinct rise in the content of glycogen (blue arrows) in the attacked muscle fiber, with membrane systems hyperplasia (X10200).

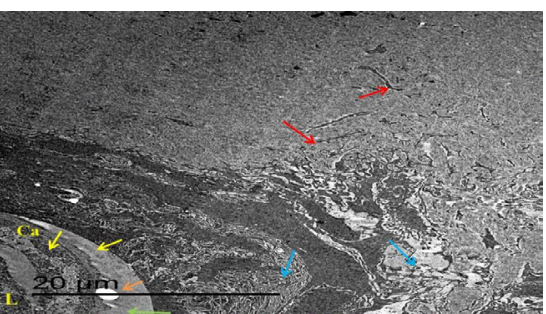

(A)

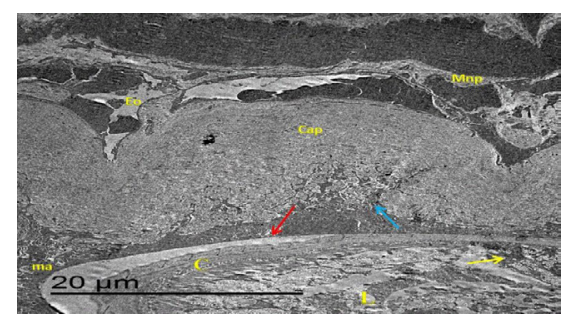

(B)

Fig. 7. TEM ultra-micrograph of diaphragm of MBZ treated G3b (X6800). A: Some calcified spots (yellow arrows) were apparent as evidence of calcification (Ca) in the larval cuticle. The matrix was partly faded and was substituted by granulomatous cells covering the calcified larva. In addition to these modifications, the matrix cytoplasm (blue arrows) often showed a significant increase in lysosomal bodies and complex cytolysomes, having loose myelin-like structures. In the muscular layer of larva (L), reduced glycogen content (red arrows) in addition to early degenerative alterations such as vacuolization (orange arrow) were observed. Notching (green arrow) was noticed in the cuticle of larva. B: Infection region appeared seriously changed. The larval edematous body (L) comprised numerous necrotic cells (yellow arrow). Glycogen was absent. The larval cuticle (C) tended to be non-intact (disintegrated) (red arrow). There was no longer a visible matrix; the necrotic residues (blue arrow) were possibly remnants of matrix structures. Mononuclear phagocytes (Mnp) besides eosinophils (EO) piled up around the outer capsule layer (Cap). 


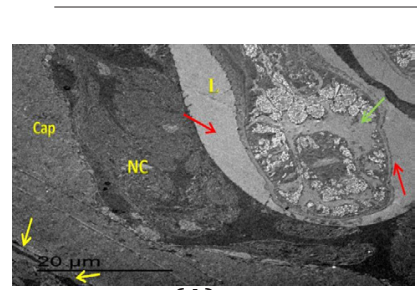

(A)

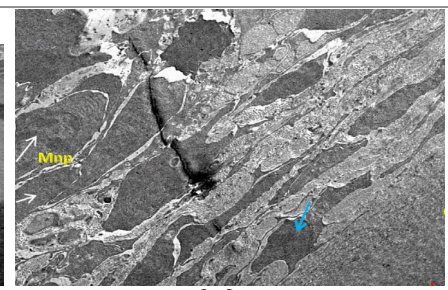

(B)

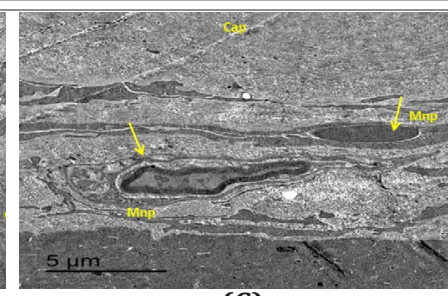

(C)

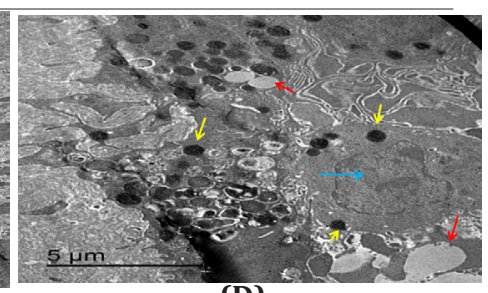

(D)

Fig. 8. TEM ultra-micrograph of diaphragm of MSCs tested G4b. A: Extremely marked vacuolization (green arrow) besides partial to complete absence of glycogen was obvious in the majority of larvae, with various degeneration signs. The cyst wall appeared separated by a gap from the larva (as it was surrounded by a trench) that showed widening of the cyst wall (red arrows), surrounded by the nurse cell (NC). Inflammatory cells settled around the capsule (yellow arrows) (X5100). B: The capsule (cap) that surrounded the larva (yellow arrow) and nurse cell (NC) (red arrow) was interrupted and disintegrated in some areas (blue arrow) and invaded by different recruited inflammatory mononuclear phagocytes (Mnp) (white arrows), lymphoid cells, eosinophils; also, to a minor degree, by mast cells and neutrophils (X10200). C: Inflammatory cells settlement in and around the capsular material and the process of inflammatory cells penetration across the capsule (cap) continued. Amongst the granuloma cells, some extremely large mononuclear phagocytes (Mnp) (yellow arrows) were found (X17000). D: Details of the nurse cell showed enormously edematous matrix that included a significant number of heterogeneous dense bodies (yellow arrows), filled up with lipid droplets (red arrows) and with disruption of mitochondria (blue arrow) (X20400). E: Calcification (blue arrows) was very pronounced, beginning at the cuticle then later on, it comprised the whole body of larva $(\mathbf{L})$. Degenerative alterations such as vacuolization were observed in the muscular layer besides the larval stichosomal cells. The disorganization of the muscular layer was commonly detected in larval body (red arrow) (X8500).

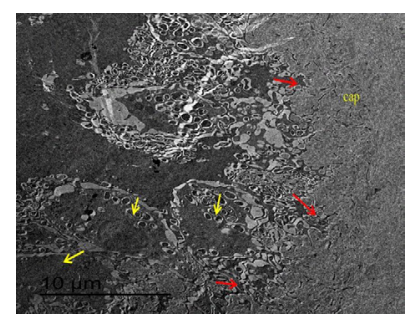

(A)

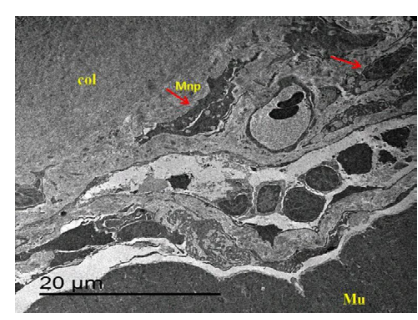

(B)

\section{DISCUSSION}

T. spiralis is a viviparous nematode and is one of the most widespread and clinically important parasites in the world. It is responsible for the disease trichinosis ${ }^{[32]}$. The pathology is characterized by inflammatory reaction in the tissues and organs, manifested by high fever, diarrhea, myalgia, periorbital edema, and serious complications as myocarditis. Reported death was due to inflammatory reaction in heart, lung and $\mathrm{CNS}^{[4]}$. Due to these adverse complications, we aimed in this study to find a new trend for treatment of $T$. spiralis, namely treatment with MSCs.

It is well known that SCs are non-specialized cells capable of indefinite differentiation to many various specialized types of cells in the body during life ${ }^{[9]}$; with the advantage of being characterized by plasticity or trans-differentiation ${ }^{[10]}$. Therapeutic SCs is a form of intervention technique that integrates new cells into damaged tissues, helping to cure several diseases and injuries ${ }^{[33,34]}$. Moreover, MSCs

Fig. 9. TEM ultra-micrograph of diaphragm of MBZ + MSCs tested G5b. A: The mononuclear phagocytes were vigorously involved in scavenging residues of matrix which was expressed by the existence of several digestive vacuoles in the interior of these cells (yellow arrows). The various constituents of both the inner and outer larva cyst walls and capsule (cap) lost their circular structure and developed a disordered fibrils network embedded inside a loose background of muscular tissue (red arrows) (X10200). B: A progressing healing stage of an infected zone evidenced by a small number of mononuclear phagocytes (Mnp) (red arrows) persisting in a region rich in collagen (col) and encircled by normal muscle fibers (Mu); nearly normal ultrastructural organization of muscle cells and interstitial space was observed (X6800).

were successfully applied in treatment of parasitic diseases like American trypanosomiasis ${ }^{[35]}$ and experimental toxoplasmosis ${ }^{[36]}$ after having been tried experimentally in schistosomiasis for both treatment ${ }^{[8]}$ and vaccination ${ }^{[37]}$. Accordingly, we were encouraged to test bone marrow MSCs as a potential line for trichinosis treatment in murine models. The delivery site of MSCs can influence the path of MSCs to reach the aimed organ. Systemic administration can be attained through IP, intravenous (IV), intraarterial, or intra-cardiac injections ${ }^{[38]}$. Braid et al. ${ }^{[39]}$ stated that engineered MSCs that were given IM were still noticeable at the insertion site more than 100 days following transplantation because of the persistent secretion of a functional antibody into circulation. Therefore, IP and IM routes of administration were chosen over IV infusion as MSCs are preserved in the insulted tissues, e.g., muscular tissues.

In the current study, MSCs were labeled using PKH26 fluorescent lipophilic dye which is referred to as "membrane dyes" and is preferred for its simplicity, and rapid effect. Also, it can be applied for 
almost any type of cells ${ }^{[40]}$. So, in both SCs monotherapy and combined therapy groups, the appearance of fluorescent dots under the fluorescent microscope, in muscular tissue sections confirmed homing of MSCs. Our results were supported by Seyed-Jafari et al. ${ }^{[41]}$ who detected migration of PKH26-treated neural SCs to the subjacent cerebral parenchyma and displayed neuronal and astrocytic cell markers. Also, MSCs have the capability of migration into the tissues from the circulation, possibly responding to signals induced by injuries. MSCs express in vitro chemotaxis towards a diversity of wound healing cytokines such as tumor necrosis factor-alpha (TNF- $\alpha$ ), platelet derived growth factor (PDGF), insulin-like growth factor-1 (IGF-1) in addition to interleukin-8 (IL-8), which clarifies their favored migration to injured sites ${ }^{[42]}$.

The current results revealed a considerable reduction in the average number of adults and larvae with the maximum effect on both intestinal and muscular phases in the combined therapy (MBZ + MSCs) groups followed by MBZ monotherapy. However, MSCs proved effective only against the muscular phase of trichinosis. The SCs proved ineffective against adult T. spiralis worms in the intestinal phase. This can be clarified by the fact that when orally administered, the SCs get digested like any other eaten cells. This explanation is also confirmed by English ${ }^{[43]}$.

During the intestinal phase, MBZ oral treatment for three successive days at a dose of $50 \mathrm{mg} / \mathrm{kg}$ body weight was effective in G3a $(\mathrm{R} \%=84.5 \%)$ and was apparently more effective when combined with MSCs (G5a) $(\mathrm{R} \%=85.5 \%)$. There was a significant difference amongst results of the two groups when compared to untreated controls. Applying the same MBZ treatment regimen, McCracken et al. ${ }^{[4]}$ similarly reported a reduction of $78 \%$ in the average number of adult worms as compared to the untreated controls.

Regarding the parasitological study results of the muscular phase, there was significant decreased number of T. spiralis larva in muscles of mice treated with MBZ, MSCs and combined treatment when compared to infected controls. The most successful treatment with significant decrease in $T$. spiralis larva number was in G5b (MBZ + MSCs) followed by G3b (MBZ) then G4b (MSCs) with percentage reductions of $91.7 \%, 87.3 \%$ and $70.0 \%$, respectively. This result confirms that MBZ is one of the most useful anthelmintics in trichinosis treatment. Its efficacy is due to its immunomodulatory actions and its capability to inhibit glycolysis and reproductive function processes in worms of $T$. spiralis $^{[45]}$. A significant finding was recorded when it was used alone (G3b) with the reduction percentage of $87.3 \%$ in larvae, supporting that MBZ is also effective in the muscular phase. This result agreed with Fernando and Denham ${ }^{[46]}$ who reported that MBZ $50 \mathrm{mg} / \mathrm{kg}$ daily for seven successive days was more effective on encysted muscle larvae than flubendazole, with reduction percentage of $99.7 \%$; while flubendazole had no effect with a reduction percentage of 3.3\%.

Considering the route of MSCs administration in our study, there was a significant decrease in the number of $T$. spiralis larvae in muscles of mice treated with IM infusion of MSCs, more than when delivered by IP injection. These results can be explained by presence of retained MSCs at the IM insertion sites, indicating that this route presents chances for regulated MSC dosing, as opposed to dynamic cell delivery as a result of IP injection amongst organs and visceral tissues. Braid et al. ${ }^{[47]}$ reinforced our findings by reporting that the IM route supported elongated cell persistence of neonatal as well as adult-derived MSCs. Therefore, IM injection offers a valuable alternative to attain clinical profits from extended MSCs settling time at a homeostatic insertion site, besides being a slightly invasive route appropriate for several applications. These results can also be clarified by the immunomodulatory effects of MSCs since Ren et $a l^{[48]}$ declared that MSCs have the capacity to control inflammatory progress and restore injured cells and tissues by adhering to areas of inflammation.

As previously reported ${ }^{[49]}$, histopathological examination of mice small intestines in the control infected G2a showed the adult worms in the lumen of the intestine, atrophied villi, minor hyperplasia of crypts, interrupted epithelial lining as well as the presence of inflammatory infiltrate in some areas. The improvements were found in MBZ treated G3a and MBZ + MSCs treated G5a that showed no adult worms, limited number of ulcers and acute inflammatory cells in the intestinal mucosa. These findings emphasized that MBZ is highly active against the intestinal phase when used alone or combined with MSCs. The current results were also partially supported by McCracken ${ }^{[50]}$. Regarding the histopathological examination of muscle tissue in infected control mice G2b, the result showed a huge number of $T$. spiralis encysted larvae that were typical in size and were firmly coiled between muscle fibers inside well-developed cysts. Muscle tissue of MBZ treated G3b showed a fewer number of $T$. spiralis larvae that were of below-average size, lightly coiled, or arc-like. The capsule was encircled and penetrated by diffuse infiltrate of inflammatory cells. Similar findings were reported by Hess et al. ${ }^{[51]}$. An improvement was found in MBZ and MSCs treated G5b that showed the highest rates of decline in the number of larvae in muscle sections.

Apoptosis is a planned cell death mechanism that stabilizes the proliferation of cells ${ }^{[52]}$. Parasites can provoke apoptosis by two means; either directly via active mediators or indirectly through the mediators of inflammation ${ }^{[53,54]}$. Previous studies explained apoptosis caused by Trichinella in infected muscle 
cells ${ }^{[55,56]}$. The histochemical assessment in our study confirms these studies where the greatest intestinal cells' DNA defense with the slightest apoptotic alterations was demonstrated as bright red coloration in the combined therapy (G5a) followed by MBZ (G3a) compared to the infected control group (G2a). The latter revealed severe apoptosis evidenced by the very faint red coloration of the nuclei. On the other hand, result of G4a showed severe apoptosis in the intestinal phase although treated with MSCs, and these results were similar to the control infected G2a. These findings may be explained by Karmańska et al. ${ }^{[57]}$ who reported that T. spiralis provoked apoptosis in the small intestine's lamina propria in striated muscle.

In the present study, stained nuclei of muscle tissue of the infected control group showed marked apoptosis with a mild red coloration of the muscle cells nuclei adjacent to larval and nuclear fragmentation. These results agreed with Etewa et al. ${ }^{[58]}$ who reported degenerative changes in experimental trichinosis with marked apoptosis in the infected control group, demonstrating the destructive influence of the parasite on muscle cells. The MBZ-treated G3b exhibited pale red coloration of muscle cells' nuclear DNA, i.e., the apoptosis was moderate since MBZ improved the majority of apoptotic alterations early. Regarding G4b and G5b, both groups revealed normal circular nuclei, and the color of their DNA was less than bright red, indicating that apoptosis was mild.

The complex "nurse cell-T. spiralis larva" is made of a changed part of a skeletal muscle cell and the larva wall. The pathological mechanism accountable for this complex formation was identified as basophilic transformation, necessary for larvae development. The alterations in the morphological structure of nurse cell-larva complex is a strong indication of immune responses elicited by tested drugs ${ }^{[59]}$. TEM images of a transformed part of $T$. spiralis infected muscle cell in mice revealed the loss of normal structures of the sarcomere besides contractile function disorganization. The filaments of actin and myosin began to fade, and the expanded smooth endoplasmic reticulum presented more or less condensed membranes structures amongst which abundant mitochondria, free ribosomes as well as vesicles of various electron density and sizes were found. Hypertrophic nuclei with nucleoli in the transcriptional phase were evident. These notifications were in agreement with Dąbrowska et al. ${ }^{[60]}$. MBZ treated G3b showed the edematous body of a larva that included different necrotic cells. The larva cuticle appeared non-intact and glycogen was no longer identified. The familiar matrix was no longer perceived, the necrotic traces were likely remains of matrix structures containing a large amount of highly expanded and disordered lamellar cytoplasm network with swollen mitochondria. Mononuclear phagocytes and eosinophils accumulated along the outer capsular layer. Regarding glycogen depletion, the present results were highlighted by De Nollin and Van den Bossche ${ }^{[61]}$ who stated that MBZ induced inhibition of the glucose uptake followed by increased glycogen depletion which may be the origin of its activity against the encysting phase of $T$. spiralis. In addition to the change in its structure, the capsule was attacked by mononuclear phagocytes, lymphoid cells, eosinophils, and to a minor degree, by neutrophils and mast cells. The mononuclear phagocytes were vigorously involved in scavenging matrix residues, and this was manifested by the existence of several digestive vacuoles inside those cells.

The most interesting observations regarding morphological changes of nurse cell-larva complex were noted in the combined MBZ and MSCs treated G5b where the progressive stages of healing were detected in the infected region. The granulomatous lesions vanished in parts, leaving only a small number of mononuclear phagocytes behind. The normal ultrastructural composition of the muscle cells and of the interstitial space was observed. The larvae had vanished totally, but some bundles of collagen persisted among the well-organized muscle fibers and calcification spots. The importance of our findings was highlighted by Nollin et al. ${ }^{[62]}$.

In conclusion, methods used for evaluation and assessment together with the present outcomes raised our interest in MSCs use as a new therapeutic line of anti-trichinosis treatment, either alone or combined with approved drugs. Additional work is needed with altered doses, routes of administration as well as various anti-trichinosis drug formulations and combinations in order to include MSCs in the anti-trichinosis therapeutic line. More work and further assessment are still needed in this aspect.

Author contribution: Sarhan MH, Etewa SE, AlHoot AA, Arafa SZ conceived and designed the research idea. Sarhan MH, Mohammad SM, Moawad HSF, Shokir RA were responsible for the acquisition, analysis, and interpretation of resulting data. Sarhan MH, Mohammad SM, Moawad HSF wrote the draft of the manuscript. Sarhan MH, and Al-Hoot AA completed the critical revision of the article. Sarhan $\mathrm{MH}$, and Etewa SE approved the final version for publication.

Conflicts of interest: On behalf of all authors, the corresponding author states that there is no conflict of interest.

Funding statement: Nil.

\section{REFERENCES}

1. García A, Leonardi D, Vasconi MD, Hinrichsen LI, Lamas MC. Characterization of albendazole-randomly methylated- $\beta$-cyclodextrin inclusion complex and in 
vivo evaluation of its antihelmitic activity in a murine model of trichinellosis. PLoS One 2014; 9(11):e113296.

2. Gottstein B, Pozio E, Nöckler K. Epidemiology, diagnosis, treatment, and control of trichinellosis. Clin Microbiol Rev 2009; 22(1):127-145.

3. Hosking BC, Watson TG, Leathwick DM. Multigeneric resistance to oxfendazole by nematodes in cattle. Vet Rec 1996; 138(3):67-68.

4. Capó V, Despommier DD. Clinical aspects of infection with Trichinella spp. Clin Microbiol Rev 1996; 9(1):47-54.

5. Aguayo-Ortiz R, Méndez-Lucio 0, Medina-Franco JL, Castillo R, Yépez-Mulia L, Hernández-Luis F, et al. Towards the identification of the binding site of benzimidazoles to $\beta$-tubulin of Trichinella spiralis: insights from computational and experimental data. J Mol Graph Model 2013; 41:12-19.

6. Lacey E. Mode of action of benzimidazoles. Parasitol Today 1990; 6(4):112-5.

7. Caner A, Döşkaya M, Değirmenci A, Can H, Baykan S, Uner A, et al. Comparison of the effects of Artemisia vulgaris and Artemisia absinthium growing in western Anatolia against trichinellosis (Trichinella spiralis) in rats. Exp Parasitol 2008; 119(1):173-179.

8. Abou Rayia DM, Elmarhoumy SM, Ismail HH, Elchennawy, FA, Zalata KR. The Outcomes of bone marrow stromal cell therapy in schistosomal hepatic fibrosis: an experimental study. J Egypt Soc Parasitol 2017; 47(3):633-642.

9. Cruz MH, Siden A, Tasat DR, Yakisich JS. Are all glioma cells cancer stem cells? J Cancer Sci Ther 2010; 2:100106.

10. Krause DS. Plasticity of marrow-derived stem cells. Gene Ther 2002; 9(11):754-758.

11. Zhang Y, Mi JY, Rui YJ, Xu YL, Wang W. Stem cell therapy for the treatment of parasitic infections: is it far away? Parasitol Res 2014; 113(2):607-612.

12. Aryamand S, Khademvatan S, Diba K, Manafpour N, Abbassi E. Stem cell therapy in the treatment of parasitic diseases. Iran J Med Microbiol 2017; 11(3):1-9.

13. Abo-Aziza FAM, Zaki AA, Abo El-Maaty AM. Bone marrowderived mesenchymal stem cell (BM-MSC): A tool of cell therapy in hydatid experimentally infected rats. Cell Regeneration 2019; 8(2):58-71.

14. Gamble HR. Detection of trichinellosis in pigs by artificial digestion and enzyme immunoassay. J Food Prot 1996; 59(3):295-298.

15. Denham DA. Studies with methyridine and Trichinella spiralis. I. Effect upon the intestinal phase in mice. Exp Parasitol 1965; 17(1):10-14.

16. Siriyasatien P, Yingyourd P, Nuchprayoon S. Efficacy of albendazole against early and late stage of Trichinella spiralis infection in mice. J Med Assoc Thai 2003; 86(Suppl2): S257-262.

17. Pulavendran S, Vignesh J, Rose C. Differential antiinflammatory and anti-fibrotic activity of transplanted mesenchymal vs. hematopoietic stem cells in carbon tetrachloride-induced liver injury in mice. Int Immunopharmacol 2010; 10(4):513-519.

18. Freshney RI. Culture of animal cells: a manual of basic technique and specialized applications, $6^{\text {th }}$ ed. 2010; John Wiley \& Sons Inc., Hoboken, New Jersey, USA.
19. Calabrò L, Fonsatti E, Bellomo G, Alonci A, Colizzi F, Sigalotti L, et al. Differential levels of soluble endoglin (CD105) in myeloid malignancies. J Cell Physiol 2003; 194:171-175.

20. Lodie TA, Blickarz CE, Devarakonda TJ, He C, Dash AB, Clarke J, et al. Systematic analysis of reportedly distinct populations of multipotent bone marrow-derived stem cells reveals a lack of distinction. Tissue Eng 2002; 8(5):739-751.

21. Louis KS, Siegel AC. Cell viability analysis using trypan blue: manual and automated methods. Methods Mol Biol 2011; 740:7-12.

22. Cho KA, Ju SY, Cho SJ, Jung YJ, Woo SY, Seoh JY, et al. Mesenchymal stem cells showed the highest potential for the regeneration of injured liver tissue compared with other subpopulations of the bone marrow. Cell Biol Int 2009; 33(7):772-777.

23. Li P, Zhang R, Sun H, Chen L, Liu F, Yao C, et al. PKH26 can transfer to host cells in vitro and vivo. Stem Cells Dev 2013; 22(2):340-344.

24. Dunn IJ, Wright KA. Cell injury caused by Trichinella spiralis in the mucosal epithelium of $\mathrm{B} 10 \mathrm{~A}$ mice. $\mathrm{J}$ Parasitol 1985; 71(6):757-766.

25. Nassef NE, El-Sobky MM, Afifi AF. Worm and larval burden, histopathological and ultrastructural evaluation of $T$. spiralis vaccination using crude worms and/or larvae antigens: Experimental studies. PUJ 2010; 3:2738.

26. Zeromski J, Boczoń K, Wandurska-Nowak E, MozerLisewska I. Effect of aminoguanidine and albendazole on inducible nitric oxide synthase (iNOS) activity in $T$. spiralis-infected mice muscles. Folia Histochem Cytobiol 2005; 43(3):157-159.

27. Monib MEM, Shaheen MS, Galal LA, Farrag HM. Role of $T$. spiralis adult and larval antigens in immunomodulation of nitric oxide (NO) in intestinal and muscular phase of trichinelosis. Assiut Med J 2010; 34:147-158.

28. Shalaby MA, Moghazy FM, Shalaby HA, Nasr SM. Effect of methanolic extract of Balanites aegyptiaca fruits on enteral and parenteral stages of Trichinella spiralis in rats. Parasitol Res 2010; 107(1):17-25.

29. Mekonnen GA, Ijzer J, Nederbragt H. Tenascin-C in chronic canine hepatitis: immunohistochemical localization and correlation with necro-inflammatory activity, fibrotic stage, and expression of alpha-smooth muscle actin, cytokeratin 7, and $\mathrm{CD}^{+}$cells. Vet Pathol 2007; 44(6):803-813.

30. Chieco P, Derenzini M. The Feulgen reaction 75 years on. Histochem Cell Biol 1999; 111(5):345-358.

31. Walski M, Owczarska K, Borowicz J. Crystalloid structures in the neuronal nuclei of the cerebral cortex of rats following total ischemia. J Hirnforsch 1995; 36(3):399-410.

32. Liu X, Song Y, Jiang N, Wang J, Tang B, Lu H, et al. Global gene expression analysis of the zoonotic parasite Trichinella spiralis revealed novel genes in host parasite interaction. PLoS Negl Trop Dis 2012; 6(8):e1794.

33. Aejaz HM, Aleem AK, Parveen N, Khaja MN, Narusu ML, Habibullah CM. Stem cell therapy-present status. Transplant Proc 2007; 39(3):694-699. 
34. Nadig RR. Stem cell therapy: Hype or hope? A review. J Conserv Dent 2009; 12(4):131-138.

35. Vilas-Boas F, Feitosa GS, Soares MB, Mota A, Pinho-Filho JA, Almeida AJ, et al. Early results of bone marrow cell transplantation to the myocardium of patients with heart failure due to Chagas disease. Arq Bras Cardiol 2006; 87(2):159-166.

36. Etewa SE, Al-Hoot AA, Abdelmoaty SM, Mohammad SM, Moawad HSF, Sarhan MH, et al. The outcomes of mesenchymal stem cells therapy for experimental toxoplasmosis. PUJ 2019; 12(1):34-44.

37. Etewa SE, Abd Allah SH, Badawey MSR, Shalaby SM, ElShal AS, El Shafey MA, et al. The effect of stem cells as an adjuvant on the immunogenicity of a potential antischistosomal vaccine in mice. J Egypt Soc Parasitol 2016; 46(3):693-716.

38. Freyman T, Polin G, Osman H, Crary J, Lu M, Cheng L, et al. A quantitative, randomized study evaluating three methods of mesenchymal stem cell delivery following myocardial infarction. Eur Heart J 2006; 27(9):11141122.

39. Braid LR, Hu WG, Davies JE, Nagata LP. Engineered mesenchymal cells improve passive pmmune protection against lethal Venezuelan equine encephalitis virus exposure. Stem Cells Transl Med 2016; 5(8):1026-1035.

40. Horan PK, Melnicoff MJ, Jensen BD, SlezakSE. Fluorescent cell labeling for in vivo and in vitro cell tracking. Methods Cell Biol 1990; 33:469-490.

41. Seyed-Jafari SS, Ali Aghaei A, Asadi-Shekaari M, Nematollahi-Mahani SN, Sheibani V. Investigating the effects of adult neural stem cell transplantation by lumbar puncture in transient cerebral ischemia. Neurosci Lett 2011; 495(1):1-5.

42. Hemeda H, Jakob M, Ludwig AK, Giebel B, Lang S, Brandau S. Interferon-gamma and tumor necrosis factor-alpha differentially affect cytokine expression and migration properties of mesenchymal stem cells. Stem Cells Dev 2010; 19(5):693-706.

43. English D. What are the benefits of consuming stem cells? https://www.quora.com/What-are-the-benefitsof-consuming-stem-cells. [Online; 2016] (last accessed 25 April 2021).

44. McCracken RO, Garcia A, Robins HG. Mebendazole therapy of enteral trichinellosis. J Parasitol 1982; 68(2):259-262.

45. Tolstoj VA, Lytvynets A, Langrova I. Pro-oxidant effects of mebendazole in albino rats experimentally infected with Trichinella spiralis. Parasitol Res 2007; 100:12771280.

46. Fernando SS, Denham DA. The effects of mebendazole and fenbendazole on Trichinella spiralis in mice. J Parasitol 1976; 62(6):874-876.

47. Braid LR, Wood CA, DWiese DM, Ford BN. Intramuscular administration potentiates extended dwell time of mesenchymal stromal cells compared to other routes. Cytotherapy 2018; 20(2):232-244.

48. Ren G, Zhang L, Zhao X, Xu G, Zhang Y, Roberts AI, et al. Mesenchymal stem cell-mediated immunosuppression occurs via concerted action of chemokines and nitric oxide. Cell Stem Cell 2008; 2(2):141-150.

49. Yu YR, Deng MJ, Lu WW, Zhang JS, Jia MZ, Huang J, et al. Endoplasmic reticulum stress-mediated apoptosis is activated in intestines of mice with Trichinella spiralis infection. Exp Parasitol 2014; 145:1-6.

50. McCracken RO. Efficacy of mebendazole and albendazole against Trichinella spiralis in mice. J Parasitol 1978. 64(2):214-219.

51. Hess JA, Chandrasekar PH, Mortiere M, Molinari JA. Comparative efficacy of ketoconazole and mebendazole in experimental trichinosis. Antimicrob Agents Chemother 1986; 30(6):953-954.

52. Osborne BA. Apoptosis and the maintenance of homoeostasis in the immune system. Curr Opin Immunol 1996; 8(2):245-254.

53. Lundy SK, Lerman SP, Boros DL. Soluble egg antigenstimulated T helper lymphocyte apoptosis and evidence for cell death mediated by FasL $\mathrm{L}^{(+)} \mathrm{T}$ and B cells during murine Schistosoma mansoni infection. Infect Immun 2001; 69(1):271-280.

54. Tato P, Fernández AM, Solano S, Borgonio V, Garrido E, Sepúlveda J, et al. A cysteine protease from Taenia solium metacestodes induce apoptosis in human CD4 ${ }^{+}$ T-cells. Parasitol Res 2004; 92(3):197-204.

55. Matsuo A, Wu Z, Nagano I, Takahashi Y. Five types of nuclei present in the capsule of Trichinella spiralis. Parasitology 2000; 121(Pt 2):203-210.

56. Boonmars T, Wu Z, Nagano I, Takahashi Y. Expression of apoptosis-related factors in muscles infected with Trichinella spiralis. Parasitology 2004; 128(Pt 3):323332.

57. Karmańska K, Houszka M, Piekarska J. The phenomenon of apoptosis in the course of experimental trichinellosis in mice. Wiad Parazytol 2000; 46(1):111-115.

58. Etewa SE, Fathy GM, Abdel-Rahman SA, El-Khalik DA, Sarhan MH, Badawey MS. The impact of anthelminthic therapeutics on serological and tissues apoptotic changes induced by experimental trichinosis. J Parasit Dis 2018; 42(2):232-242.

59. Wu Z, Sofronic-Milosavljevic Lj, Nagano I, Takahashi Y. Trichinella spiralis: nurse cell formation with emphasis on analogy to muscle cell repair. Parasit Vectors 2008; 1(1):27.

60. Dąbrowska J, Walski M, Machnicka B, Grytner-Zięcina B. TEM studies on morphological "junctions" between Trichinella spiralis larvae and mouse skeletal muscle cells with particular emphasis on the early changes in host muscles. Acta Parasit 2008; 53:411-414.

61. De Nollin S, Van den Bossche H. Biochemical effects of mebendazole on Trichinella spiralis larvae. J Parasitol 1973; 59(6): 970-976.

62. Nollin SD, Borgers M, Vanparijs O, Van Den Bossche $\mathrm{H}$. Effects of mebendazole on the encysted phase of Trichinella spiralis in the rat: an electron microscope study. Parasitology 1974; 69(1):55-62. 